\title{
Metformin inhibits growth of human non-small cell lung cancer cells via liver kinase B-1-independent activation of adenosine monophosphate-activated protein kinase
}

\author{
QIANQIAN GUO $^{1 *}$, ZHIYAN LIU $^{2 *}$, LILI JIANG $^{1}$, MENGJIE LIU $^{1}$, JIEQUN MA $^{1}$, \\ CHENGCHENG YANG $^{1}$, LILI HAN ${ }^{1}$, KEJUN NAN $^{1}$ and XUAN LIANG ${ }^{1}$ \\ ${ }^{1}$ Department of Oncology, First Affiliated Hospital, Xi'an Jiaotong University Medical College, Xi'an, \\ Shaanxi 710061; ${ }^{2}$ Department of Respiration, Xi'an Central Hospital, Xi'an, Shaanxi 710003, P.R. China
}

Received March 8, 2015; Accepted January 11, 2016

DOI: $10.3892 / \mathrm{mmr} .2016 .4830$

\begin{abstract}
Metformin, the most widely administered oral anti-diabetic therapeutic agent, exerts its glucose-lowering effect predominantly via liver kinase B1 (LKB1)-dependent activation of adenosine monophosphate-activated protein kinase (AMPK). Accumulating evidence has demonstrated that metformin possesses potential antitumor effects. However, whether the antitumor effect of metformin is via the LKB1/AMPK signaling pathway remains to be determined. In the current study, the effects of metformin on proliferation, cell cycle progression, and apoptosis of human non-small cell lung cancer (NSCLC) H460 (LKB1-null) and H1299 (LKB1-positive) cells were assessed, and the role of LKB1/AMPK signaling in the anti-growth effects of metformin were investigated. Cell viability was determined using a 3-(4,5-dimethylthiazol-2-yl)-2,5-diphenyltetrazolium bromide assay, cell cycle distribution and apoptosis were assessed by flow cytometry, and protein expression levels were measured by western blotting. Metformin inhibited proliferation, induced significant cell cycle arrest at the $\mathrm{G}_{0}-\mathrm{G}_{1}$ phase and increased apoptosis in NSCLC cells in a time- and concentration-dependent manner, regardless of the level of LKB1 protein expression. Furthermore, knockdown of LKB1 with short hairpin RNA (shRNA) did not affect the antiproliferative effect of metformin in the H1299
\end{abstract}

Correspondence to: Professor Kejun Nan or Dr Xuan Liang, Department of Oncology, First Affiliated Hospital, Xi'an Jiaotong University Medical College, 277 Yanta West Road, Xi'an, Shaanxi 710061, P.R. China

E-mail: nankj1958@163.com

E-mail: elva_0209@126.com

*Contributed equally

Key words: metformin, non-small cell lung cancer, liver kinase B1, adenosine monophosphate-activated protein kinase, mammalian target of rapamycin cells. Metformin stimulated AMPK phosphorylation and subsequently suppressed the phosphorylation of mammalian target of rapamycin and its downstream effector, 70-kDa ribosomal protein S6 kinase in the two cell lines. These effects were abrogated by silencing AMPK with small interfering RNA (siRNA). In addition, knockdown of AMPK with siRNA inhibited the effect of metformin on cell proliferation in the two cell lines. These results provide evidence that the growth inhibition of metformin in NSCLC cells is mediated by LKB1-independent activation of AMPK, indicating that metformin may be a potential therapeutic agent for the treatment of human NSCLC.

\section{Introduction}

Metformin is an oral biguanide agent that is widely administered as a first line therapy to patients with diabetes mellitus type 2 (1). Metformin improves hyperglycemia, predominantly by reducing hepatic glucose production via inhibition of gluconeogenesis, and enhancing glucose uptake and utilization by reversal of insulin resistance in peripheral tissues $(2,3)$. It has been confirmed that the glucose-lowering action of metformin is reliant on liver kinase B1 (LKB1)-dependent activation of adenosine monophosphate-activated protein kinase (AMPK) $(4,5)$. AMPK is an evolutionarily conserved cellular energy sensor, which is activated in response to an increased AMP/adenosine triphosphate (ATP) ratio caused by various cellular stresses, including starvation. Once activated, AMPK phosphorylates a variety of downstream substrates resulting in the restoration of cellular energy levels by activating ATP-generating processes, while deactivating ATP-consuming processes (6).

It has been well documented that AMPK activation is required for metformin to inhibit glucose production in hepatocytes and stimulate glucose uptake in isolated rat skeletal muscles (5). LKB1, a major upstream serine/threonine kinase of AMPK, is also required for the glucose-lowering action of metformin, as the deletion of LKB1 in the liver was demonstrated to eliminate the effect of metformin on serum glucose levels in mice (4). Notably, LKB1 was initially identified as a tumor suppressor responsible for an inherited 
cancer susceptibility disease, Peutz-Jeghers syndrome (7). Dysfunction of the LKB1 tumor suppressor is important in cancer development (8). The LKB1/AMPK-dependent glucose-lowering functions of metformin have stimulated investigation into the anti-tumor effects of metformin (9).

Epidemiological studies have demonstrated that metformin administration is associated with a reduction in cancer risk and cancer-associated mortality in patients with diabetes $(10,11)$, which indicates the potential antitumor efficacy of metformin. Subsequently, increasing evidence has shown that metformin significantly inhibits the growth of various cancer cells in vitro and in vivo, including in breast, prostate and gastric cancer, and lymphoma (12-15). Since the glucose-lowering action of metformin primarily depends on the LKB1/AMPK signaling pathway, it is proposed that the antitumor effect of metformin also utilizes this signaling pathway. Indeed, metformin selectively inhibited proliferation of LKB1-positive breast cancer cells, which was accompanied by activation of AMPK (13); cancer cells with dysfunctional LKB1 were not inhibited and downregulation of AMPK with small interfering RNA (siRNA) prevented metformin-induced growth inhibition in the cells (13). However, it was also reported that metformin inhibited proliferation of LKB1-null cancer cells (16). Furthermore, Ben Sahra et al (17) identified that metformin induced cell-cycle arrest by inhibiting mammalian target of rapamycin (mTOR) activity independently of AMPK. Therefore, the role of the LKB1/AMPK signaling pathway in the antineoplastic effect of metformin remains controversial.

Prospective studies have demonstrated that preoperative administration of metformin suppresses the growth of cancer cells in breast and endometrial cancers $(18,19)$, which provide direct evidence that metformin inhibits malignant growth. Lung cancer is the most common type of malignant tumor and also the leading cause of cancer-associated mortality worldwide, with non-small cell lung cancer (NSCLC) accounting for $\sim 80 \%$ (20). It is highly possible that patients with NSCLC may also benefit from the anti-diabetic therapeutic agent, metformin. However, notably, $\leq 30 \%$ of NSCLC patients exhibit functional loss of LKB1 (21), which may limit the application of metformin for the treatment of NSCLC. Due to the high mutation frequency of LKB1 in NSCLC, it is necessary to elucidate the role of the LKB1/AMPK signaling pathway regarding the antineoplastic effect of metformin in NSCLC. In the present study, the effects of metformin on the growth of cultured NSCLC H460 and H1299 cells were investigated, and whether the LKB1/AMPK signaling pathway mediates the antitumor effect of metformin in NSCLC cells was evaluated.

\section{Materials and methods}

Cell lines and culture. Human H1299 and H460 NSCLC cell lines were purchased from the cell bank of the Shanghai Institute of Cell Research (Shanghai, China). The two cell lines were cultured in RPMI-1640 medium (Hyclone; GE Healthcare Life Sciences, Logan, UT, USA) supplemented with $10 \%$ fetal bovine serum (TransGen Biotech, Inc., Beijing, China) and maintained in a humid atmosphere with $5 \% \mathrm{CO}_{2}$ at $37^{\circ} \mathrm{C}$.
Chemicals and antibodies. Metformin was purchased from Sigma-Aldrich (St. Louis, MO, USA) and dissolved in sterile phosphate-buffered saline (PBS; TransGen Biotech, Inc.,) at a stock concentration of $1 \mathrm{~mol} / \mathrm{l}$. The metformin was stored at $-20^{\circ} \mathrm{C}$ and diluted to the necessary concentration prior to each experiment. The primary antibodies against phosphorylated (p)-AMPK $\alpha$ and AMPK $\alpha$ were purchased from Cell Signaling Technology, Inc. (Boston, MA, USA). Primary antibodies against p-mTOR, mTOR, p-70-kDa ribosomal protein S6 kinase (p70S6K) and p-p70S6K were purchased from Bioworld Technology,Inc. (St.Louis Park, MN, USA). Primary antibodies against $\beta$-actin, and horseradish peroxidase-conjugated goat anti-mouse and goat anti-rabbit secondary antibodies were purchased from TransGen Biotech, Inc.

Transfection of siRNA and short hairpin RNA (shRNA). Cells were seeded at $2.5 \times 10^{5}$ cells/well in 6 -well plates. After $24 \mathrm{~h}$, siRNA-negative control (si-NC) and AMPK specific siRNA (si-AMPK; GenePharma Co., Ltd., Shanghai, China) were transfected into cells using Turbofect Transfection Reagent (Thermo Fisher Scientific, Inc., Waltham, MA, USA). The siRNA sequence for AMPK $\alpha$ was as follows: Forward, 5'-GCGUGUACGAAGGAAGAAUTT-3' and reverse, 5'-AUU CUUCCUUCGUACACGCTT-3'. Lentiviral vectors carrying shRNA targeting LKB1 (sh-LKB1) or shRNA-NC (sh-NC) (Genechem Co., Ltd., Shanghai, China) were transfected into cells according to the manufacturer's protocol.

Cell viability assay. Cell viability was determined using the 3-(4,5-dimethylthiazol-2-yl)-2,5-diphenyltetrazolium bromide (MTT) assay. Cells were seeded at $4 \times 10^{3}$ cells/well in 96-well plates. After $24 \mathrm{~h}$, cells were treated with $0,5,10$ or $20 \mathrm{mM}$ metformin for 24,48 and $72 \mathrm{~h}$. At the respective time-points, $20 \mu \mathrm{l}$ MTT solution (5 mg/ml; KeyGen Biotech, Nanjing, China) was added to the wells and cells were cultured for an additional $4 \mathrm{~h}$. The culture medium was removed and $150 \mu \mathrm{l}$ dimethylsulfoxide (MP Biomedicals, LLC., Santa Ana, CA, USA) was added to dissolve formazan. Cell viability was quantified by measuring absorbance at $492 \mathrm{~nm}$ using a microplate spectrophotometer (680; Bio-Rad Laboratories, Inc., Hercules, CA, USA) to calculate the optical density values.

Cell cycle analysis. Cell cycle analysis was performed using the Cell Cycle Detection kit (KeyGen Biotech) according to the manufacturer's protocol. Briefly, cells were treated with 0,5 or $10 \mathrm{mM}$ metformin for $24 \mathrm{~h}$, harvested with trypsin (TransGen Biotech, Inc.) and fixed with $70 \%$ ethanol (Guanghua Sci-Tech Co., Ltd., Shantou, China) at $4^{\circ} \mathrm{C}$ overnight. Following centrifugation at $104 \mathrm{xg}$ for $5 \mathrm{~min}$ and washed twice with PBS, cells were incubated with RNase A (KeyGen Biotech, Inc.) at $37^{\circ} \mathrm{C}$ for $30 \mathrm{~min}$ and stained with propidium iodide (PI; KeyGen Biotech, Inc.) at $4^{\circ} \mathrm{C}$ for $30 \mathrm{~min}$. DNA content was determined using a flow cytometer (FACSCanto, BD Biosciences, San Jose, CA, USA), and data were analyzed by ModFit LT software (version 3.3.11; Verity Software House, Inc., Topsham, ME, USA).

Analysis of apoptosis. Cells were harvested following treatment with 0,5 or $10 \mathrm{mM}$ metformin for $48 \mathrm{~h}$ and flow 
A

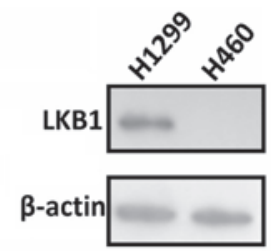

B
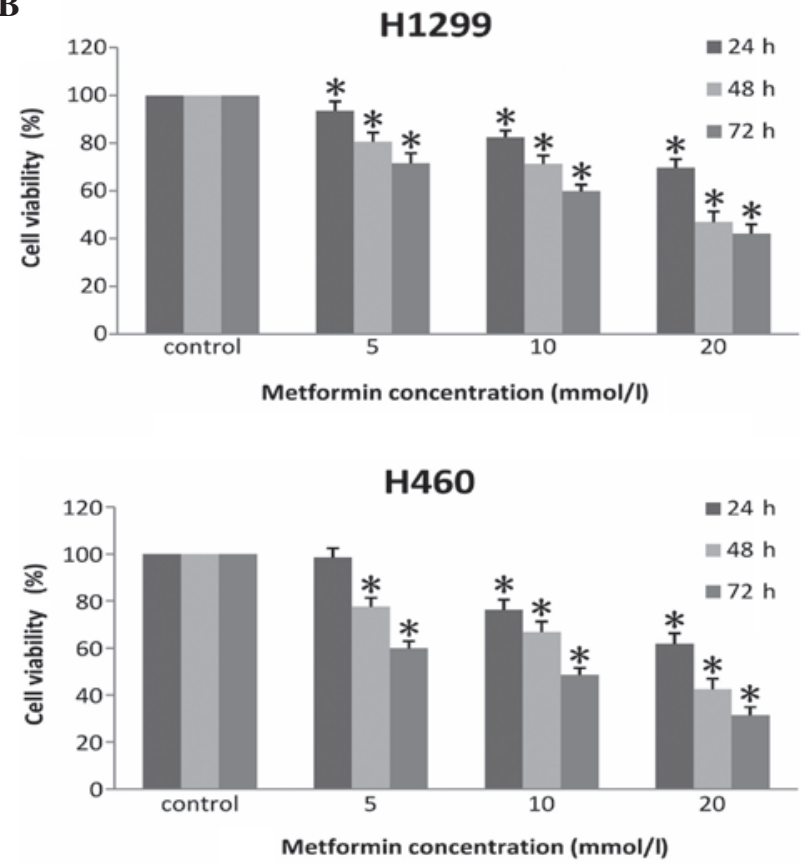

Figure 1. Metformin inhibited proliferation of human non-small cell lung cancer cells. (A) The protein expression of LKB1 in H1299 and H460 cells was analyzed by western blotting. $\beta$-actin served as a loading control. (B) H1299 and H460 cells were seeded at $4 \times 10^{3}$ cells/well in 96-well plates. After $24 \mathrm{~h}$, the culture medium was replaced with fresh culture medium containing $0,5,10$ or $20 \mathrm{mM}$ metformin for 24,48 and $72 \mathrm{~h}$. Cell viability was determined by 3-(4,5-dimethylthiazol-2-yl)-2,5-diphenyltetrazolium bromide assay at the indicated time-points. Data from three independent experiments are presented as the mean \pm standard error of the mean. ${ }^{*} \mathrm{P}<0.05$ vs. the control. LKB1, liver kinase B1.

cytometry was performed to analyze apoptosis using the Annexin V-fluorescein isothiocyanate/PI Apoptosis kit (BD Biosciences) according to the manufacturer's protocol.

Western blot analysis. Cells were lysed in radioimmunoprecipitation assay lysis buffer (Sigma-Aldrich) containing a protease and phosphatase inhibitor cocktail on ice for $30 \mathrm{~min}$. The protein lysate was collected by centrifugation at $14,500 \mathrm{xg}$ for $20 \mathrm{~min}$ at $4^{\circ} \mathrm{C}$. Equal quantities of total protein were separated by electrophoresis for $110 \mathrm{~min}$ at $100 \mathrm{~V}$ on a $10 \%$ SDS-PAGE (Sigma-Aldrich), transferred to polyvinylidene fluoride membranes (EMD Millipore, Boston, MA, USA) and incubated overnight at $4^{\circ} \mathrm{C}$ with the corresponding primary AMPK $\alpha$ polyclonal rabbit anti-human (1:1,000; cat. no. 2532), p-AMPK $\alpha$ polyclonal rabbit anti-human (1:1,000; cat. no. 2532),p-mTOR polyclonal rabbit anti-human (1:750; cat. no. BS4706), p70S6K polyclonal rabbit anti-human (1:800; cat. no. BS3633), p-p70S6K polyclonal rabbit anti-human (1:500; cat. no. BS4440) and $\beta$-actin monoclonal mouse anti-human (1:10,000; cat no. HC201) antibodies followed by incubation with the secondary
A H1299
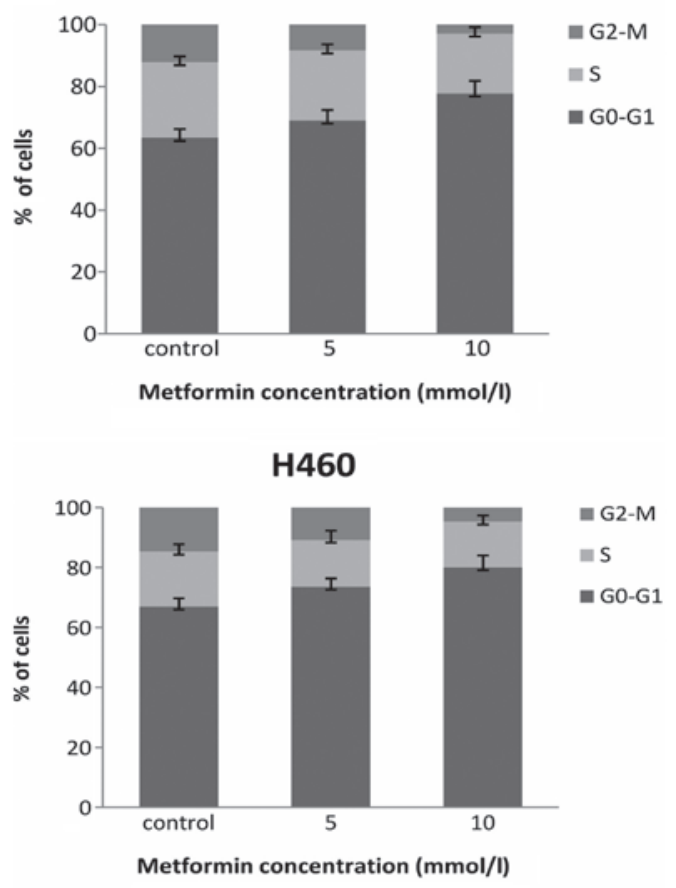

B

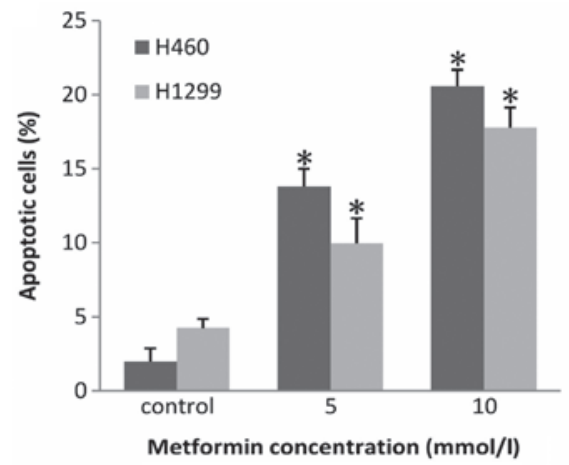

Figure 2. Metformin arrested the cell cycle at the $\mathrm{G}_{0}-\mathrm{G}_{1}$ phase, and induced apoptosis in the H460 and H1299 cells. (A) Cells were seeded in 6-well plates ( $3 \times 10^{5}$ cells/well), incubated for $24 \mathrm{~h}$, exposed to 0,5 or $10 \mathrm{mM}$ metformin for another $24 \mathrm{~h}$, and were subjected to flow cytometry for analysis of cell cycle distribution. (B) Cells were subjected to flow cytometry to analyze apoptosis following treatment with 0,5 or $10 \mathrm{mM}$ metformin for $48 \mathrm{~h}$. Data from three independent experiments are presented as the mean \pm standard error of the mean. ${ }^{*} \mathrm{P}<0.05$ vs. the control.

horseradish peroxidase-conjugated goat anti-rabbit(1:10,000; cat. no HS101) and horseradish peroxidase-conjugated goat anti-mouse (1:10,000; cat. no. HS201) antibodies for $2 \mathrm{~h}$ at room temperature. Proteins of interest were visualized using enhanced chemiluminescence kit (EMD Millipore). The band intensities were quantified by densitometry using ImageJ software (version 1.49; National Institutes of Health, Bethesda, MA, USA).

Statistical analysis. All experiments were repeated independently a minimum of three times. Data are presented as the mean \pm standard error of the mean. Statistical analyses were performed by one-way analysis of variance coupled with a Student-Newman-Keuls post hoc test using SPSS 13.0 statistical software (SPSS, Inc., Chicago, IL, USA). P<0.05 
was considered to indicate a statistically significant difference.

\section{Results}

Metformin inhibits proliferation of LKB1-null H460 and LKB1-positive H1299 cells. To investigate whether LKB1 participates in the antitumor effect of metformin, the NSCLC cell lines, H460 and H1299 were selected and the protein expression of LKB1 was verified using western blotting. As shown in Fig. 1A, LKB1 was detected in H1299 cells, but not in the H460 cells, which was consistent with previous reports $(22,23)$. The $\mathrm{H} 460$ and $\mathrm{H} 1299$ cells were treated with 5,10 or $20 \mathrm{mM}$ metformin for 24, 48 and $72 \mathrm{~h}$, and cell viability was assessed by MTT assay. Compared with the untreated control, metformin significantly inhibited proliferation of H1299 cells in a time- and concentration-dependent manner (Fig. 1B). Notably, a similar inhibitory effect was also observed in the H460 cells, which do not express LKB1. These results demonstrate that metformin was capable of inhibiting the proliferation of NSCLC cells independently of LKB1, and indicate that LKB1 is not essential for the antiproliferative effect of metformin in NSCLC cells.

Metformin induces cell cycle arrest and apoptosis in $\mathrm{H460}$ and H1299 cells. To investigate the possible mechanisms of grow th inhibition by metformin, cell cycle distribution and apoptosis were analyzed by flow cytometry in H460 and H1299 cells following treatment with metformin. Metformin treatment resulted in the delayed entry into $\mathrm{S}$ phase and a significant $\mathrm{G}_{0}-\mathrm{G}_{1}$ phase arrest in $\mathrm{H} 1299$ cells. Specifically, 5 and $10 \mathrm{mM}$ metformin increased the percentage of cells in the $G_{0}-G_{1}$ phase by 5.6 and $14.4 \%$, respectively. Similarly, the percentage of cells in the $G_{0}-G_{1}$ phase was also markedly increased in $\mathrm{H} 460$ cells following treatment with metformin (Fig. 2A). Furthermore, metformin treatment induced significant apoptosis in the two cell lines (Fig. 2B). Compared with the control, $10 \mathrm{mM}$ metformin increased the percentage of apoptotic cells by 17.53 and $15.53 \%$ in H460 and H1299 cells, respectively. Therefore, metformin induced significant cell cycle arrest at the $\mathrm{G}_{0}-\mathrm{G}_{1}$ phase and increased apoptosis in H460 and H1299 cells, regardless of LKB1 expression.

The antiproliferative effect of metformin is not mediated by $L K B 1$. To further support the observation that LKB1 was not essential for the antiproliferative effect of metformin, LKB1 was knocked down in $\mathrm{H} 1299$ cells using sh-LKB1. sh-LKB1 reduced the protein level of LKB1, when compared with sh-NC (Fig. 3A). Knockdown of LKB1 promoted cell proliferation in H1299 cells, although it did not affect the loss of cell viability following metformin treatment (Fig. 3B). These results indicate that the antiproliferative effect of metformin is independent of LKB1 in NSCLC cells.

Metformin activates AMPK in H460 and H1299 cells. To investigate whether the antiproliferative effect of metformin on H460 and H1299 cells was mediated through the AMPK signaling pathway, the effects of metformin on the phosphorylation of AMPK and its downstream target, mTOR were determined via western blot analysis. mTOR is
A

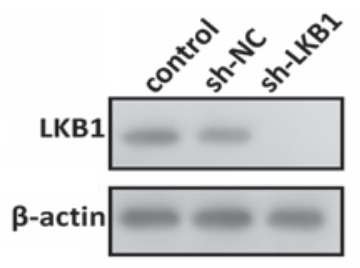

B

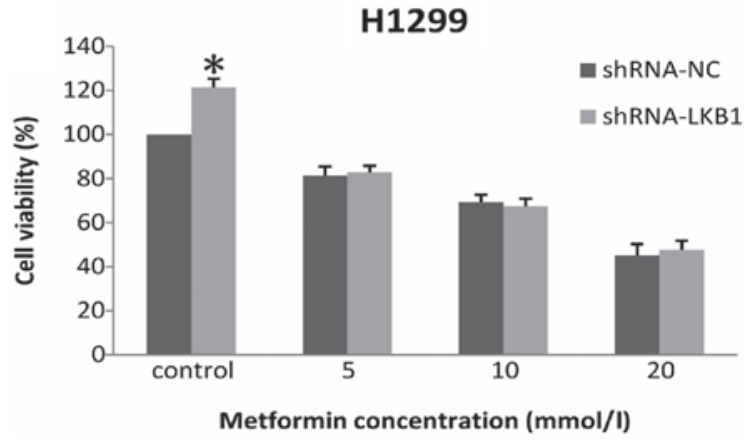

Figure 3. Silencing LKB1 did not alter the antiproliferative effects of metformin. (A) The protein expression level of LKB1 was reduced in H1299 cells following transfection with lentiviral vectors carrying shRNA-LKB1. $\beta$-actin served as a loading control. (B) H1299 cells were treated with $0,5,10$ or $20 \mathrm{mM}$ metformin for $48 \mathrm{~h}$ after transfection with shRNA-LKB1 or shRNA-NC, and a 3-(4,5-dimethylthiazol-2-yl)-2,5-diphenyltetrazolium bromide assay was performed to assess cell viability. Data from three independent experiments are presented as the mean \pm standard error of the mean. ${ }^{*} \mathrm{P}<0.05$ vs. the shRNA-NC group. LKB1, liver kinase B1; sh, short hairpin; NC, negative control.

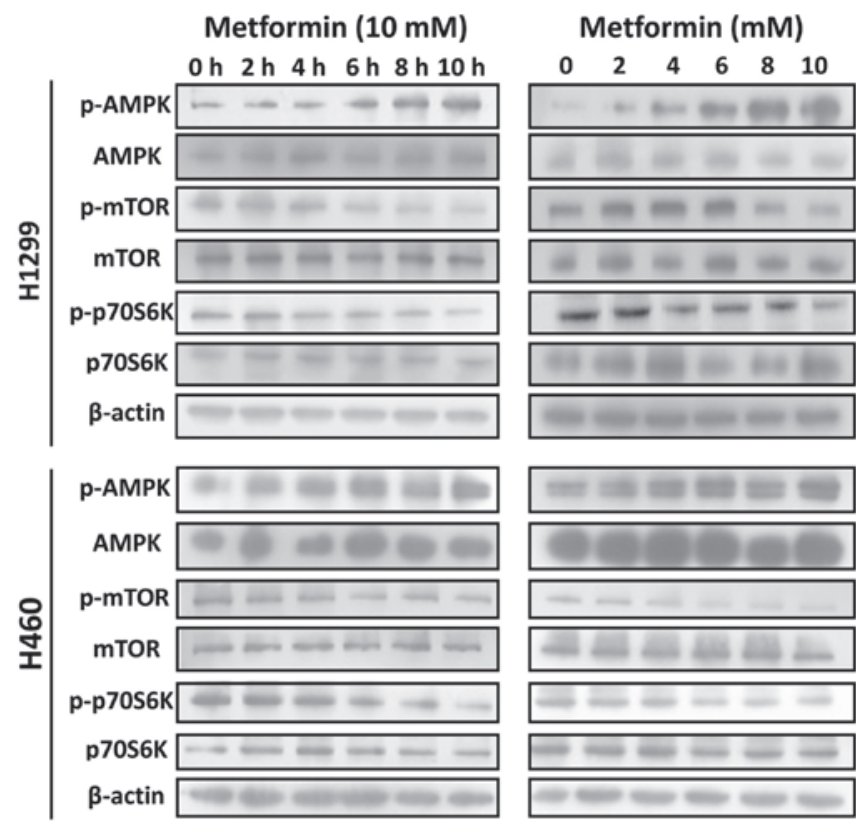

Figure 4. Metformin activated AMPK and inhibited the mTOR signaling pathway in $\mathrm{H} 460$ and $\mathrm{H} 1299$ cells. The H460 and H1299 cell lines were treated with $10 \mathrm{mM}$ metformin for the indicated times or treated with the indicated concentrations of metformin for $6 \mathrm{~h}$. Following treatment, protein extracts were examined by western blot for p-AMPK, AMPK, p-mTOR, mTOR, p-p70S6K, p70S6K and $\beta$-actin protein expression levels. Data are representative of a minimum of three independent experiments. p, phosphorylated; AMPK, adenosine monophosphate-activated protein kinase; mTOR, mammalian target of rapamycin; p70S6K, 70-kDa ribosomal protein S6 kinase.

negatively regulated by AMPK and is particularly important in regulating cell metabolism and cell growth via activating 
A

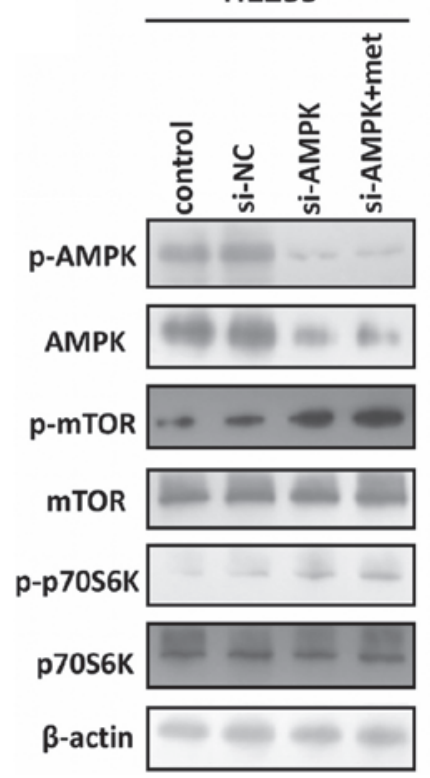

H460

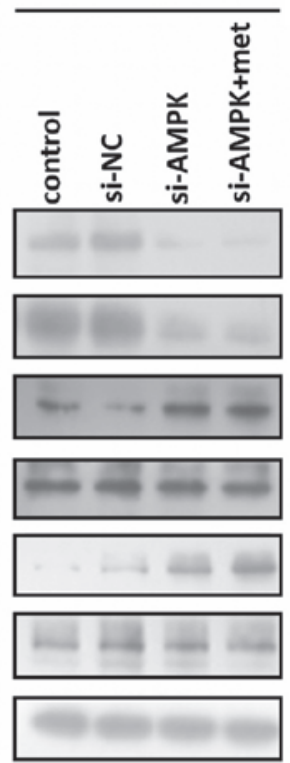

B

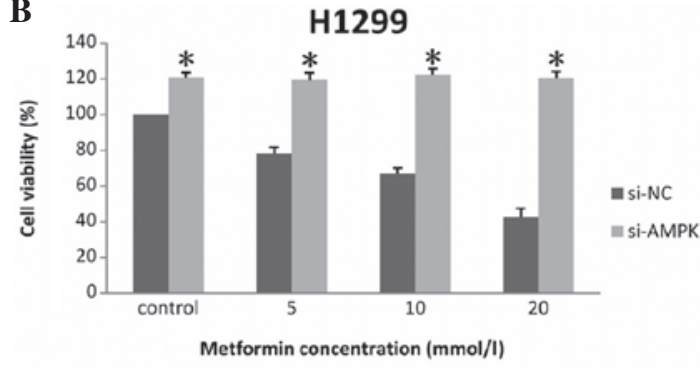

H460

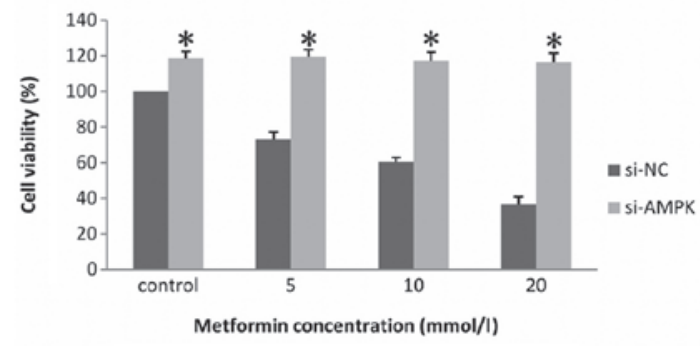

Figure 5. Knockdown of AMPK with siRNA reversed the effects of metformin on non-small cell lung cancer cells. (A) Cells were treated with 10 mM metformin for $6 \mathrm{~h}$ after transfection with si-AMPK, and examined by western blot for p-AMPK, AMPK, p-mTOR, mTOR, p-p70S6K, p70S6K and $\beta$-actin protein expression levels. Data are representative of a minimum of three independent experiments. (B) Cells were transfected with si-AMPK or si-NC. Following transfection, at $24 \mathrm{~h}$, cells were treated with $0,5,10$ or $20 \mathrm{mM}$ metformin for $48 \mathrm{~h}$, and a 3-(4,5-dimethylthiazol-2-yl)-2,5-diphenyltetrazolium bromide assay was performed to assess cell viability. Data from three independent experiments are presented as the mean \pm standard error of the mean. $\mathrm{P}<0.05$ vs. the si-NC group. p, phosphorylated; AMPK, adenosine monophosphate-activated protein kinase; met, metformin; mTOR, mammalian target of rapamycin; p70S6K, 70-kDa ribosomal protein S6 kinase; si, small interfering; NC, negative control.

various downstream effectors, including p70S6K (24). As shown in Fig. 4, metformin increased the phosphorylation of AMPK, and suppressed phosphorylation of mTOR and p70S6K in H1299 cells in a time- and concentration-dependent manner when compared with the control group. However, the total protein expression levels of AMPK, mTOR and p70S6K were not markedly altered. Similar effects were also observed in LKB1-null H460 cells. These results demonstrated that metformin activated AMPK and subsequently suppressed mTOR signaling independently of LKB1.

The antiproliferative effect of metformin is dependent on $A M P K$ activation. The mTOR signaling pathway is central in cell growth, proliferation, metabolism and survival. The activation of AMPK, and the subsequent suppression of mTOR signaling, suggests that the antiproliferative effect of metformin may depend on AMPK. To test this hypothesis, AMPK was knocked down by siRNA in the H1299 and H460 cells and the effects of metformin on mTOR activity and cell proliferation were examined. AMPK siRNA reduced the protein level of AMPK and phosphorylation of AMPK. Consistent with the negative regulation of mTOR signaling by AMPK, knockdown of AMPK by siRNA led to upregulation of the phosphorylation of mTOR and p70S6k. Furthermore, silencing AMPK abrogated metformin-induced phosphorylation of AMPK, and inhibition of mTOR and p70S6k phosphorylation (Fig. 5A). As expected, in cells transfected with control siRNA, metformin inhibited the proliferation of the two cell lines in a concentration-dependent manner. By contrast, in the H1299 and H460 cells, silencing AMPK using siRNA abolished the inhibitory effect of metformin on cell viability (Fig. 5B). These results indicate that activation of AMPK is essential for the antiproliferative effect of metformin in NSCLC cells.

\section{Discussion}

In the present study, metformin was shown to significantly inhibit cell proliferation, and induce apoptosis and cell cycle arrest at the $\mathrm{G}_{0}-\mathrm{G}_{1}$ phase in LKB1-null $\mathrm{H} 460$ and LKB1-positive H1299 NSCLC cells. Notably, the effect of metformin on growth inhibition was identified to be dependent on the activation of AMPK, but not LKB1. The results indicate that metformin inhibits growth of NSCLC cells independently of LKB1 status, and suggest that metformin is a potential agent for the treatment of NSCLC, $30 \%$ of which contain non-functional LKB1.

As a safe, efficient and inexpensive therapeutic agent, metformin has been administered to treat patients with diabetes mellitus type 2 since the 1950s (25). The glucose-lowering action of metformin depends on LKB1-dependent activation of AMPK, which inhibits hepatic glucose production and promotes glucose uptake in muscles $(4,5)$. In addition to its anti-diabetic effect, the anticancer efficacy of metformin has become a point of interest; epidemiology studies have reported a decrease in cancer risk and cancer-associated mortality in diabetic patients treated with metformin $(10,11)$. Furthermore, increasing evidence has demonstrated that metformin is capable of inhibiting the growth of various cancer cells in vitro and in vivo (12-15). The antitumor action of metformin is associated with induction of cell cycle arrest, apoptosis or autophagy $(12,14-17,26)$, however, the underlying molecular mechanisms are not completely understood. Although it is widely accepted that the glucose lowering effect of metformin 
is mediated via the LKB1/AMPK signaling pathway, to the best of our knowledge, the role of LKB1/AMPK in the antitumor effect of metformin has not yet been fully determined.

LKB1, a serine/threonine kinase, phosphorylates 14 protein kinases, including AMPK $\alpha 1$, AMPK $\alpha 2$ and 12 AMPK-related kinases (ARKs). As the roles of ARKs remain unknown, it is proposed that LKB1 functions predominantly through activating AMPK (27). The primary function of the LKB1/AMPK signaling pathway is the regulation of cell metabolism and cell growth (27). It has been reported that metformin inhibits proliferation and protein translation in LKB1-positive breast cancer cells, while these effects were not observed in LKB1-null cancer cells (13). Furthermore, Rattan et al (28) reported that downregulation of LKB1 with specific siRNA attenuated metformin-induced activation of AMPK and reversed the antiproliferative action of metformin in ovarian cancer cells. These observations led to the conclusion that the antitumor effect of metformin relies on functional LKB1. By contrast, Algire et al (29) proposed that cancer cells with dysfunctional LKB1 were more sensitive to metformin. Notably, the current results demonstrated that metformin inhibited growth of LKB1-positive H1299 cells to a similar level of LKB1-null H460 cells. Furthermore, metformin continued to inhibit cell proliferation of H1299 cells following silencing of LKB1 with shRNA. These results suggest that LKB1 is not essential for the anti-growth effect of metformin and dysfunctional LKB1 does not impair the antitumor effect of metformin in NSCLC.

Promotion of apoptosis and induction of cell cycle arrest are the major antitumor mechanisms of numerous chemotherapeutic agents (30). Metformin has been shown to block the cell cycle at the $\mathrm{G}_{0}-\mathrm{G}_{1}$ phase in various types of cancer cell $(12,15,17,29,31)$. Consistent with these data, the present results revealed that NSCLC cells, regardless of the expression level of LKB1, were arrested at the $G_{0}-G_{1}$ phase in response to metformin treatment. Previous studies have also reported that metformin promotes apoptosis in certain cancer cells, including pancreatic cancer, melanoma and oral squamous cell carcinoma $(16,31,32)$. However, Ben Sahra et al (15) reported that metformin did not induce apoptosis in prostate cancer cells. The flow cytometry results from the present study demonstrate that metformin markedly induced apoptosis in the H460 and H1299 NSCLC cells. This discrepancy concerning the effect of metformin on apoptosis may result from specificity of various cancer cells and differences in experimental conditions, indicating that further experiments are required to define the proapoptotic action of metformin. Recent studies have demonstrated that metformin induces autophagy, mitochondrial damage and oxidative stress in melanoma cells $(16,26)$. This indicates that the antitumor mechanisms of metformin are not restricted to the induction of apoptosis and cell cycle arrest. Thus, it remains to be investigated whether metformin induces these cellular processes in NSCLC cells.

AMPK is a cellular energy sensor and is activated in response to cellular energy stress. Activated AMPK phosphorylates crucial metabolic enzymes, which switch on catabolic pathways (that generate ATP) and switch off ATP-consuming anabolic pathways, thereby restoring the cellular energy level (6). In addition to its metabolic regulation,
AMPK is important in tumorigenesis. It has been reported that AMPK activity is decreased in human lung cancer tissue samples (33), and AMPK activation leads to cell cycle arrest and growth inhibition in cancer cells (34). It is well established that metformin lowers serum glucose via activation of AMPK. However, it is not clear whether the antitumor action of metformin relies on AMPK activation. Zakikhani et al (13) reported that metformin inhibited the growth of breast cancer cells via activation of AMPK. Similar results were reported in ovarian cancer cells (28). However, certain studies proposed that the inhibitory effect of metformin on cell growth does not require activation of AMPK $(17,26)$. Consistent with the former conclusions, the present results demonstrated that AMPK is essential for metformin to inhibit growth of NSCLC cells. Firstly, metformin activated AMPK in the two types of NSCLC cell lines that were evaluated, and this was associated with increased phosphorylation of AMPK, and decreased phosphorylation of mTOR and p70S6K. Secondly, AMPK siRNA attenuated metformin-induced inhibition of the mTOR pathway and reversed the antiproliferative action of metformin.

The discrepancy among studies concerning the role of the LKB1/AMPK signaling pathway in the antitumor effect of metformin may be due to the cell specificity, as well as the complexity of mechanisms that are mediated by metformin. The direct target of metformin is neither LKB1 nor AMPK, but complex I of the mitochondrial respiratory chain $(35,36)$. Through inhibition of complex I, metformin activates AMPK in cancer and normal cells via at least two mechanisms. First, the inhibition of complex I by metformin leads to the phosphorylation and activation of protein kinase $\mathrm{C}(\mathrm{PKC})-\zeta$. Activated PKC- $\zeta$ phosphorylates LKB1 at Ser428, resulting in LKB1 nuclear export and subsequent AMPK activation (37). Second, the interruption of the mitochondrial respiratory chain by metformin directly affects the generation of ATP and causes an increase in the cellular AMP/ATP ratio, which activates AMPK in an LKB1-independent manner. Ouyang et al (38) reported that the mechanism involves inhibition of AMP deaminase, rather than activation of LKB1; whereby, metformin activates AMPK and stimulates glucose uptake in skeletal muscle cells. Furthermore, metformin has been reported to exert its glucose-lowering and antitumor effects via multiple AMPK-independent mechanisms (17,26,39). In the present study, it was demonstrated that the anti-growth effect of metformin in NSCLC cells was dependent on LKB1-independent activation of AMPK. Metformin significantly inhibited proliferation, blocked cell cycle progression and induced apoptosis in the H460 and H1299 NSCLC cells regardless of LKB1 expression. Knockdown of LKB1 with shRNA did not alter the antiproliferative effect of metformin in the H1299 cells. In addition, metformin activated AMPK, and knockdown of AMPK rescued cells from the growth inhibitory effect of metformin.

In conclusion, the data from the present study revealed that metformin significantly inhibited cell proliferation, induced apoptosis and blocked cell cycle progression in NSCLC cells. Notably, the antitumor effect of metformin required activation of AMPK, but not LKB1. These results provide novel insights into the action of metformin on NSCLC cells, and suggest that metformin may be a potential candidate for novel therapeutic strategies for the treatment of human NSCLC. 


\section{Acknowledgements}

The present study was supported by the Social Development and Scientific and Technological Research Project of Shaanxi province (grant no. 2013k12-08-3), and the Special Assistance Fund for Clinical Research of Wu Jieping Medical Foundation (grant no. 320.6799.1128).

\section{References}

1. [No authors listed] Standards of medical care in diabetes - 2015 Summary of revisions. Diabetes Care 38 (Suppl 4): 2015.

2. Sarabia V, Lam L, Burdett E, Leiter LA and Klip A: Glucose-transport in human skeletal-muscle cells in culture. Stimulation by insulin and metformin. J Clin Invest 90: 1386-1395, 1992.

3. Hundal RS, Krssak M, Dufour S, Laurent D, Lebon V, Chandramouli V, Inzucchi SE, Schumann WC, Petersen KF, Landau BR and Shulman GI: Mechanism by which metformin reduces glucose production in type 2 diabetes. Diabetes 49: 2063-2069, 2000.

4. Shaw RJ, Lamia KA, Vasquez D, Koo SH, Bardeesy N, DePinho RA, Montminy M and Cantley LC: The kinase LKB1 mediates glucose homeostasis in liver and therapeutic effects of metformin. Science 310: 1642-1646, 2005.

5. Zhou GC, Myers R, Li Y, Chen Y, Shen X, Fenyk-Melody J, Wu M, Ventre J, Doebber T, Fujii N, et al: Role of AMP-activated protein kinase in mechanism of metformin action. J Clin Invest 108: 1167-1174, 2001.

6. Kahn BB, Alquier T, Carling D and Hardie DG: AMP-activated protein kinase: Ancient energy gauge provides clues to modern understanding of metabolism. Cell Metab 1: 15-25, 2005.

7. Hemminki A, Markie D, Tomlinson I, Avizienyte E, Roth S, Loukola A, Bignell G, Warren W, Aminoff M, Höglund P, et al A serine/threonine kinase gene defective in Peutz-Jegheus syndrome. Nature 391: 184-187, 1998.

8. Sanchez-Cespedes M: A role for LKB1 gene in human cancer beyond the Peutz-Jeghers syndrome. Oncogene 26: 7825-7832, 2007.

9. Ben Sahra I, Le Marchand-Brustel Y, Tanti JF and Bost F: Metformin in cancer therapy: A new perspective for an old antidiabetic drug? Mol Cancer Ther 9: 1092-1099, 2010

10. Landman GW, Kleefstra N, van Hateren KJ, Groenier KH, Gans RO and Bilo HJ: Metformin associated with lower cancer mortality in Type 2 Diabetes - ZODIAC-16. Diabetes Care 33: 322-326, 2010

11. Evans JM, Donnelly LA, Emslie-Smith AM, Alessi DR and Morris AD: Metformin and reduced risk of cancer in diabetic patients. BMJ 330: 1304-1305, 2005.

12. Kato K, Gong J, Iwama H, Kitanaka A, Tani J, Miyoshi H, Nomura K, Mimura S, Kobayashi M, Aritomo Y, et al: The antidiabetic drug metformin inhibits gastric cancer cell proliferation in vitro and in vivo. Mol Cancer Ther 11: 549-560, 2012.

13. Zakikhani M, Dowling R, Fantus IG, Sonenberg N and Pollak M: Metformin is an AMP kinase-dependent growth inhibitor for breast cancer cells. Cancer Res 66: 10269-10273, 2006.

14. Shi WY, Xiao D, Wang L, Dong LH, Yan ZX, Shen ZX, Chen SJ, Chen Y and Zhao WL: Therapeutic metformin/AMPK activation blocked lymphoma cell growth via inhibition of mTOR pathway and induction of autophagy. Cell Death Dis 3: e275, 2012.

15. Ben Sahra I, Laurent K, Loubat A, Giorgetti-Peraldi S, Colosetti P, Auberger P, Tanti JF, Le Marchand-Brustel Y and Bost F: The antidiabetic drug metformin exerts an antitumoral effect in vitro and in vivo through a decrease of cyclin D1 level. Oncogene 27: 3576-3586, 2008.

16. Tomic T, Botton T, Cerezo M, Robert G, Luciano F, Puissant A, Gounon P, Allegra M, Bertolotto C, Bereder JM, et al: Metformin inhibits melanoma development through autophagy and apoptosis mechanisms. Cell Death Dis 2: e199, 2011.

17. Ben Sahra I, Regazzetti C, Robert G, Laurent K, Le Marchand-Brustel Y, Auberger P, Tanti JF, Giorgetti-Peraldi S and Bost F: Metformin, independent of AMPK, induces mTOR inhibition and cell-cycle arrest through REDD1. Cancer Res 71: 4366-4372, 2011.
18. Mitsuhashi A, Kiyokawa T, Sato Y and Shozu M: Effects of metformin on endometrial cancer cell growth in vivo: A preoperative prospective trial. Cancer 120: 2986-2995, 2014.

19. Niraula S, Dowling RJ, Ennis M, Chang MC, Done SJ, Hood N, Escallon J, Leong WL, McCready DR, Reedijk M, et al: Metformin in early breast cancer: A prospective window of opportunity neoadjuvant study. Breast Cancer Res Treat 135: 821-830, 2012.

20. Siegel R, Naishadham D and Jemal A: Cancer statistics, 2013. CA-Cancer J Clin 63: 11-30, 2013.

21. Matsumoto S, Iwakawa R, Takahashi K, Kohno T, Nakanishi Y, Matsuno Y, Suzuki K, Nakamoto M, Shimizu E, Minna JD and Yokota J: Prevalence and specificity of LKB1 genetic alterations in lung cancers. Oncogene 26: 5911-5918, 2007.

22. Sanchez-Cespedes M, Parrella P, Esteller M, Nomoto S, Trink B, Engles JM, Westra WH, Herman JG and Sidransky D: Inactivation of LKB1/STK11 is a common event in adenocarcinomas of the lung. Cancer Res 62: 3659-3662, 2002.

23. Carretero J, Medina PP, Pio R, Montuenga LM and Sanchez-Cespedes M: Novel and natural knockout lung cancer cell lines for the LKB1/STK11 tumor suppressor gene. Oncogene 23: 4037-4040, 2004.

24. Guertin DA and Sabatini DM: Defining the role of mTOR in cancer. Cancer Cell 12: 9-22, 2007.

25. Duncan LJ and Seaton DA: The treatment of diabetes mellitus with metformin. Br J Clin Pract 16: 129-132, 1962.

26. Janjetovic K, Harhaji-Trajkovic L, Misirkic-Marjanovic M, Vucicevic L, Stevanovic D, Zogovic N, Sumarac-Dumanovic M, Micic D and Trajkovic V: In vitro and in vivo anti-melanoma action of metformin. Eur J Pharmacol 668: 373-382, 2011.

27. Hardie DG and Alessi DR: LKB1 and AMPK and the cancer-metabolism link-ten years after. BMC Biol 11: 36, 2013.

28. Rattan R, Giri S, Hartmann LC and Shridhar V: Metformin attenuates ovarian cancer cell growth in an AMP-kinase dispensable manner. J Cell Mol Med 15: 166-178, 2011.

29. Algire C, Amrein L, Bazile M, David S, Zakikhani M and Pollak M: Diet and tumor LKB1 expression interact to determine sensitivity to anti-neoplastic effects of metformin in vivo. Oncogene 30: 1174-1182, 2011.

30. Waldman T, Zhang Y, Dillehay L, Yu J, Kinzler K, Vogelstein B and Williams J: Cell-cycle arrest versus cell death in cancer therapy. Nat Med 3: 1034-1036, 1997.

31. Luo Q, Hu D, Hu S, Yan M, Sun Z and Chen F: In vitro and in vivo anti-tumor effect of metformin as a novel therapeutic agent in human oral squamous cell carcinoma. BMC Cancer 12: $517,2012$.

32. Wang LW, Li ZS, Zou DW, Jin ZD, Gao J and Xu GM: Metformin induces apoptosis of pancreatic cancer cells. World J Gastroenterol 14: 7192-7198, 2008

33. Conde E, Suarez-Gauthier A, García-García E, Lopez-Rios F, Lopez-Encuentra A, García-Lujan R, Morente M, Sanchez-Verde L and Sanchez-Cespedes M: Specific pattern of LKB1 and phospho-acetyl-CoA carboxylase protein immunostaining in human normal tissues and lung carcinomas. Hum Pathol 38: 1351-1360, 2007.

34. Motoshima H, Goldstein BJ, Igata M and Araki E: AMPK and cell proliferation-AMPK as a therapeutic target for atherosclerosis and cancer. J Physiol 574: 63-71, 2006.

35. El-Mir MY, Nogueira V, Fontaine E, Avéret N, Rigoulet M and Leverve X: Dimethylbiguanide inhibits cell respiration via an indirect effect targeted on the respiratory chain complex I. J Biol Chem 275: 223-228, 2000.

36. Owen MR, Doran E and Halestrap AP: Evidence that metformin exerts its anti-diabetic effects through inhibition of complex 1 of the mitochondrial respiratory chain. Biochem J 348: 607-614, 2000.

37. Xie Z, Dong Y, Scholz R, Neumann D and Zou MH: Phosphorylation of LKB1 at serine 428 by protein kinase C-zeta is required for metformin-enhanced activation of the AMP-activated protein kinase in endothelial cells. Circulation 117: 952-962, 2008.

38. Ouyang JY, Parakhia RA and Ochs RS: Metformin activates AMP kinase through inhibition of AMP deaminase. J Biol Chem 286: 1-11, 2011.

39. Foretz M, Hébrard S, Leclerc J, Zarrinpashneh E, Soty M, Mithieux G, Sakamoto K, Andreelli F and Viollet B: Metformin inhibits hepatic gluconeogenesis in mice independently of the LKB1/AMPK pathway via a decrease in hepatic energy state. J Clin Invest 120: 2355-2369, 2010. 